\title{
Analysis of Anharmonic EXAFS Spectra of Crystalline Nickel Using High-order Debye-Waller Factors
}

\author{
Tong Sy Tien ${ }^{1, *}$, Le Viet Hoang", Nguyen Ngoc Thang', Bui Ba Manh ${ }^{3}$, Nguyen Huu Hieu³, \\ Nguyen Thi Ngoc Anh ${ }^{3}$, Duong Thanh Cong ${ }^{4}$, Nguyen Hong Nhung ${ }^{4}$, Nguyen Thi Thanh Nhan ${ }^{5}$ \\ ${ }^{1}$ Institute of Research \& Development, Duy Tan University, Danang, Vietnam \\ ${ }^{2}$ Department of Physics, Hanoi University of Science, Hanoi, Vietnam \\ ${ }^{3}$ Department 2, University of Fire, Hanoi, Vietnam \\ ${ }^{4}$ Department 10, University of Fire, Hanoi, Vietnam \\ ${ }^{5}$ Department 1, University of Fire, Hanoi, Vietnam
}

Email address:

tongsytien@duytan.edu.vn (T. Sy Tien)

${ }^{*}$ Corresponding author

\section{To cite this article:}

Tong Sy Tien, Le Viet Hoang, Nguyen Ngoc Thang, Bui Ba Manh, Nguyen Huu Hieu, Nguyen Thi Ngoc Anh, Duong Thanh Cong, Nguyen Hong Nhung, Nguyen Thi Thanh Nhan. Analysis of Anharmonic EXAFS Spectra of Crystalline Nickel Using High-order Debye-Waller Factors. Advances in Applied Sciences. Vol. 5, No. 3, 2020, pp. 70-74. doi: 10.11648/j.aas.20200503.13

Received: June 25, 2020; Accepted: July 14, 2020; Published: August 5, 2020

\begin{abstract}
The extended X-ray absorption fine structure (EXAFS) has been developed into a powerful technique and is widely applied to determine many structural parameters and dynamic properties of materials. The EXAFS technique is now the technique of choice in many materials science investigations, and the EXAFS data analysis is being performed in many laboratories spread around the world. In this work, the anharmonic EXAFS spectra of crystalline nickel (Ni) has been analyzed based on the quantum anharmonic correlated Einstein model. The anharmonic EXAFS oscillation presented in terms of the Debye-Waller factors using the cumulant expansion approach up to the fourth-order. This calculation model has been developed from the high-order anharmonic effective potential that described the contribution of their nearest-neighbor atoms to the pair interaction potential. The analytical expressions of the anharmonic EXAFS cumulants are not only explicit forms but also satisfy all of their fundamental properties in temperature dependence. The analysis of the anharmonic EXAFS spectra was performed by evaluating the contributions of the cumulants to the amplitude reduction and the phase shift of the anharmonic EXAFS oscillation. The numerical results for $\mathrm{Ni}$ were in good agreement with those obtained using the other theoretical methods and experiment at various temperatures, which are useful for analyzing the experimental EXAFS data of the metal crystals.
\end{abstract}

Keywords: EXAFS Analysis, Einstein Model, Quantum Statistical Theory, Crystalline Nickel

\section{Introduction}

The structural parameters and dynamic properties of materials can be determined from the extended X-ray absorption fine structure (EXAFS) technique [1-5]. The use of the moments of the radial distribution function (or cumulants) to investigate local disorder of EXAFS spectra was introduced by Rehr (1979) [10] who showed that the Debye-Waller (DW) factors of EXAFS spectra has a natural cumulant expansion in powers of the photoelectron wavenumber. The connection between the Debye-Waller (DW) factors and the EXAFS cumulants was described in detail in the cumulant expansion approach (ratio method) by Bunker (1983) [7] and exploited by Tranquada \& Ingalls (1983) [11]. The ratio method is particularly appealing because it summarizes the relevant structural and dynamic information that easily obtained from the experimental EXAFS spectra [8, 9, 12-14]. However, the position of atoms is not stationary, and their interatomic distance always changes due to thermal vibrations [4-6] that were detected by Beni \& Platzman (1976) [2] and Eisenberger \& Brown (1979) [3]. They cause anharmonic effects on crystal vibrations and smear out the EXAFS oscillations $[3,6]$. The 
anharmonicity of the potential yields additional terms in the EXAFS oscillation $[3,5,10]$, so if ignoring these terms, it can lead to non-negligible errors in the structural parameters [11-15].

Many efforts have been performed to calculate the cumulants for including anharmonic effects in the EXAFS spectra by many various approaches such as the full lattice dynamic approach [16], the path-integral effective-potential method [17, 18], the equation of motion method [19], the path-integral Monte Carlo calculation [20, 21], and the density functional theory calculation [22, 23]. The anharmonic EXAFS oscillation is expanded as a series of cumulants of the interatomic distance distribution, which is effectively achieved in analyzing experimental EXAFS spectra [8, 11, 12]. Although each approach has certain limitations, the importance of including higher-order cumulants in the anharmonic EXAFS analysis has been recognized in many works [8, 11-13, 17, 24].

Recently, the quantum anharmonic correlated Einstein (QACE) model using the first-order perturbation was developed by Tien (2020) [25]. It calculates the first four EXAFS cumulants and analyzes the EXAFS amplitude and phase of FCC crystal. It has also been applied to successfully investigate crystalline copper $(\mathrm{Cu})$ successfully but has not yet investigated crystalline nickel (Ni). Therefore, the analysis of the anharmonic EXAFS spectra of Ni by using the QACE model will be a necessary addition to evaluate the effectiveness of the QACE model. The purpose of this work is to calculate and estimate the role of the EXAFS cumulants in analyzing the anharmonic EXAFS oscillation of $\mathrm{Ni}$ by using the QACE model.

\section{Basic Formulae of EXAFS Function}

The EXAFS oscillation for a single coordination shell, including thermal disorders has the form:

$$
\chi(k, T)=A(k, T) \sin \Phi(k, T),
$$

where $A(k, T)$ and $\Phi(k, T)$ are the EXAFS amplitude and phase, respectively, and $k$ is the photoelectron wavenumber [6-8], and $T$ is the temperature.

The $K$-edge EXAFS oscillation for the distribution of identical atoms is described within the framework of singlescattering and plane-wave approximations [7, 8, 11, 12]. Following the approach from Ref. 25, the logarithm of amplitude ratio $M\left(k, T_{1}, T_{2}\right)=\ln \left[A\left(k, T_{2}\right) / A\left(k, T_{1}\right)\right]$ and the linear phase difference $\Delta \Phi\left(k, T_{1}, T_{2}\right)=\Phi\left(k, T_{2}\right)-\Phi\left(k, T_{1}\right)$ between temperatures $T_{2}$ and $T_{1}$ in the cumulant expansion approach up to the fourth order, which are given as follows:

$$
\begin{gathered}
M\left(k, T_{1}, T_{2}\right) \simeq-2 k^{2}\left\{\sigma^{2}\left(T_{2}\right)-\sigma^{2}\left(T_{1}\right)\right\}+\frac{2 k^{4}}{3}\left\{\sigma^{(4)}\left(T_{2}\right)-\sigma^{(4)}\left(T_{1}\right)\right\}, \\
\Delta \Phi\left(k, T_{1}, T_{2}\right) \simeq 2 k\left\{\sigma^{(1)}\left(T_{2}\right)-\sigma^{(1)}\left(T_{1}\right)\right\}-4 k\left\{\frac{1}{r_{0}}+\frac{1}{\lambda}\right\}\left\{\sigma^{2}\left(T_{2}\right)-\sigma^{2}\left(T_{1}\right)\right\}-\frac{4 k^{3}}{3}\left\{\sigma^{(3)}\left(T_{2}\right)-\sigma^{(3)}\left(T_{1}\right)\right\} .
\end{gathered}
$$

where $\sigma^{(n)}$ are $n$ th-order cumulants and can be expressed in terms of the power moments of the true $\mathrm{RD}$ function $\rho(T, r)$.

To determine the thermodynamic parameters of a system, it is necessary to specify its anharmonic effective potential and force constants $[15,20,24,25]$. For metal crystals, the anharmonic effective (AE) potential was used effectively in many works [26-30]. In the relative vibrations of absorbing $(A)$ and backscattering $(B)$ atoms, including correlation effects and taking into account only the nearest-neighbor interactions, the AE model [15] is given by

$$
V_{e f f}=V(x)+\sum_{i=A, B} \sum_{j \neq A, B} V\left(\frac{\mu}{M_{i}} x \hat{R}_{A B} \hat{R}_{i j}\right),
$$

where $\mu=M_{A} M_{B} /\left(M_{A}+M_{B}\right)$ is the reduced mass of the absorber and backscatterer with masses $M_{A}$ and $M_{B}$, respectively, $\hat{R}$ is a unit vector, the sum $i$ is the over absorbers $(i=A)$ and backscatterers $(i=B)$, and the $\operatorname{sum} j$ is over the nearest neighbors.

The Morse potential was proposed by Girifalco and Weizer (1959) [31] to calculate the efficiency of the interaction energy between each pair of atoms in cubic metals. The Morse potential was expanded up to the fourth-order in the form as

$$
V(x)=D\left(e^{-2 \alpha x}-2 e^{-\alpha x}\right) \cong-D+D \alpha^{2} x^{2}-D \alpha^{3} x^{3}+\frac{7}{12} D \alpha^{4} x^{4}
$$

where $x$ is the same previously defined value, $\alpha$ describes the width of the potential, and $D$ is the dissociation energy.

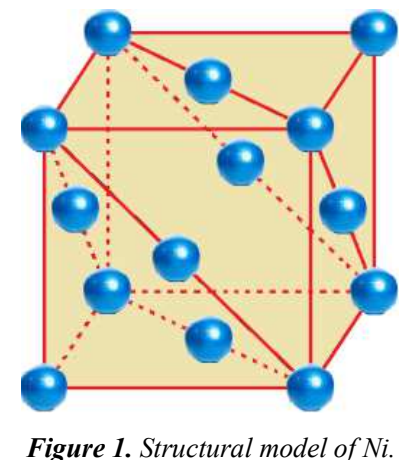

Applying Eqs. (4) and (5) to the structure of $\mathrm{Ni}$ with a mass of atoms is $M_{A}=M_{B}=m$, as seen in Figure 1, the AE potential is written as 


$$
V_{\text {eff }}(x)=\frac{5}{2} D \alpha^{2} x^{2}-\frac{5}{4} D \alpha^{3} x^{3}+\frac{133}{192} \alpha^{4} x^{4} .
$$

The local force constants $k_{0}, k_{3}$, and $k_{4}$ are deduced from Eq. (6) as follows:

$$
k_{0}=5 D \alpha^{2}, \quad k_{3}=\frac{5}{4} D \alpha^{3}, \quad k_{4}=\frac{133}{192} D \alpha^{4} .
$$

The thermal vibration of atoms is characterized by the correlated Einstein frequency $\omega_{E}$ and temperature $\theta_{E}$, which are calculated from the effective force constant $k_{0}$ in the following forms:

$$
\omega_{E}=\sqrt{\frac{k_{e f f}}{\mu}}=\alpha \sqrt{\frac{10 D}{m}}, \theta_{E}=\frac{\hbar \omega_{E}}{k_{B}}=\frac{\hbar \alpha}{k_{B}} \sqrt{\frac{10 D}{m}},
$$

where $k_{B}$ is the Boltzmann constant, $\hbar$ is the reduced Planck constant.

Consequently, the correlated Einstein frequency $\omega_{E}$ and temperature $\theta_{E}$, and the force constants $k_{0}, k_{3}$, and $k_{4}$ are

expressed in terms of the Morse potential parameters via Eqs. (7) and (8).

\section{Temperature Dependence of EXAFS Cumulants Within the QACE Model}

The EXAFS cumulants are explicitly related to low-order moments of the distribution function [32]. The analysis of the EXAFS spectra usually uses the first four EXAFS cumulants $[8,10,12,33]$. The expressions of the first four cumulants within the QACE model are given as follows [25]:

$$
\begin{gathered}
\sigma^{(1)}=\frac{3 \hbar \omega_{E}}{40 D \alpha}\left(\frac{1+z}{1-z}\right), \\
\sigma^{2}=\frac{\hbar \omega_{E}}{10 D \alpha^{2}}\left(\frac{1+z}{1-z}\right), \\
\sigma^{(3)}=\frac{\hbar^{2} \omega_{E}^{2}}{200 D^{2} \alpha^{3}} \cdot \frac{1+10 z+z^{2}}{(1-z)^{2}},
\end{gathered}
$$

$$
\sigma^{(4)}=\frac{\hbar^{3} \omega_{E}^{3}}{160000 D^{3} \alpha^{4}} \cdot \frac{(1+z)\left(947+1096 z+947 z^{2}\right)}{(1-z)^{3}}-\frac{399 \hbar^{4} \omega_{E}^{4}}{40000 D^{3} \alpha^{4} k_{B} T} \cdot \frac{z^{2}}{(1-z)^{4}} .
$$

where the parameter $z=e^{-\theta_{E} / T}$, the calculations of the EXAFS cumulants use the local force constants $k_{0}, k_{3}$, and $k_{4}$, and the correlated Einstein temperature $\theta_{E}$ are determined from in Eqs. (7) and (8).

Thus, the results of the first four EXAFS cumulants are calculated using the QACE model can satisfy all of their fundamental properties in temperature dependence. These obtained results can describe both the influence of quantum effects on the vibrational energy at low temperatures and the influence of anharmonic effects on the classical limit at high temperatures.

\section{Numerical Results and Discussions}

To discuss the effectiveness of the QACE model for the analysis of the EXAFS spectra of $\mathrm{Ni}$ in this work, we apply Eqs. (2)-(3) in Sec. 2 and Eqs. (9)-(12) in Sec. 3 to the numerical calculations for $\mathrm{Ni}$. Our results are compared with those obtained using the ACD model [34], the path-integral effectivepotential (PIEP) method was developed to solve the path integral approximately by means of the variational concept [17]. Our results are also compared with the experimental values obtained by Pirog et al. (2002) [35] at the Synchrotron Radiation Siberian Center, Russia, and obtained by Yokoyama et al. (1998) [17] at Beamline 10B in the Photon Factory of the Institute of Materials Structure Science, Tsukuba, Japan. From these comparisons, we evaluate and comment on the results obtained using the QACE model in this work.
Table 1. The thermodynamic parameters $k_{0}, k_{3}, k_{4}, \omega_{E}$, and $\theta_{E}$ of $N i$ obtained using the QACE model and experiment [35].

\begin{tabular}{llllll}
\hline Method & $\begin{array}{l}\boldsymbol{k}_{0} \\
\left(\mathbf{e V} \AA^{-2}\right)\end{array}$ & $\begin{array}{l}\boldsymbol{k}_{3} \\
\left(\mathbf{e V} \AA^{-3}\right)\end{array}$ & $\begin{array}{c}\boldsymbol{k}_{4} \\
\left(\mathbf{e V} \AA^{-4}\right)\end{array}$ & $\begin{array}{c}\omega_{\boldsymbol{E}}(\times \\
\left.\mathbf{1 0}^{13} \mathbf{H z}\right)\end{array}$ & $\begin{array}{c}\boldsymbol{\theta}_{\boldsymbol{E}} \\
(\mathbf{K})\end{array}$ \\
\hline CACE model $^{\mathrm{a}}$ & 4.2 & 1.5 & 1.2 & 3.7 & 284.3 \\
Experiment $^{\mathrm{b}}$ & 3.9 & 1.6 & 1.7 & 3.6 & 272.7 \\
\hline${ }^{\mathrm{a}}$ This work. & & & & & \\
${ }^{\mathrm{b}}$ Reference 35. & & & & &
\end{tabular}
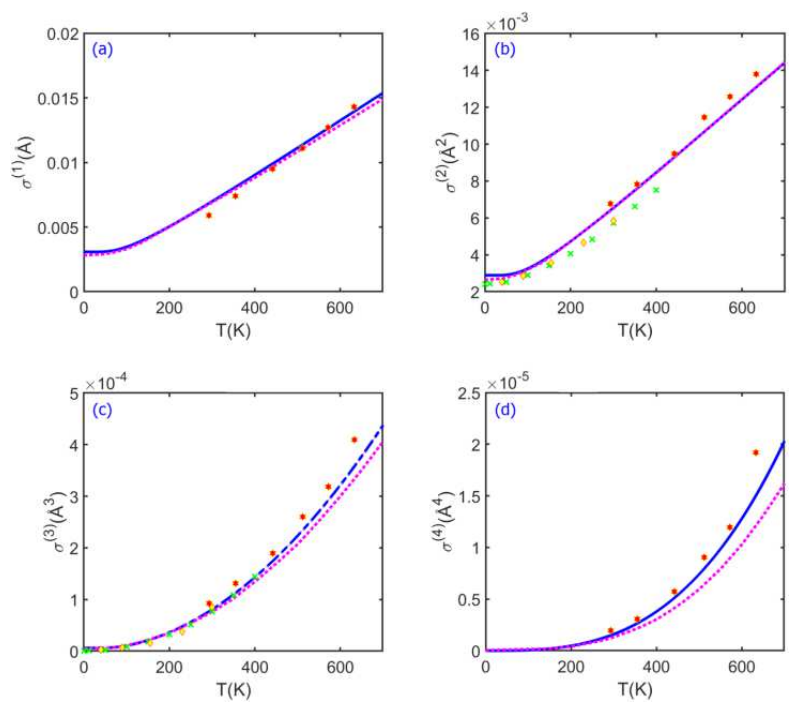

Figure 2. Temperature dependence of (a) the first, (b) second, (c) third, and (d) fourth EXAFS cumulants of Ni obtained using the QACE model (solid blue lines), the ACD model [34] (dotted magenta lines), the PIEP method [17] (green multiplication signs), and the other experiments [17] (full yellow diamonds) and [35] (full red hexagrams). 
The thermodynamic parameters $k_{0}, k_{3}, k_{4}, \omega_{E}$, and $\theta_{E}$ of $\mathrm{Ni}$ are calculated using the Morse potential parameters $D=0.4205 \mathrm{eV}$ and $\alpha=1.4199 \AA^{-1}$ [31] via Eqs. (7)-(8) and given in Table 1. These calculations. Our results agree with those obtained from the experiment [35], especially for the force constants $k_{0}$ and $k_{3}$, and the correlated Einstein frequency $\omega_{E}$ and temperature $\theta_{E}$.

The temperature dependence of (a) the first cumulant $\sigma^{(1)}(T)$, (b) the second cumulant $\sigma^{2}(T)$, (c) the third cumulant $\sigma^{(3)}(T)$, and (d) the fourth cumulant $\sigma^{(4)}(T)$ of $\mathrm{Ni}$ is calculated by Eqs. (9)-(12) and shown in Figure 2. Our obtained results using the QACE model agree well with the results obtained using the ACD model [34], the PIEP method (for the second and third cumulants) [17], and the other experiments [17] (for the second and third cumulants) and [35]. Compared with the experiments [17, 35], our results agreed better than the obtained results using the ACD model [34], especially for high-order cumulants (the third and fourth cumulants) and at high temperatures. For example, at $300 \mathrm{~K}$, the results obtained using the CACE model, the ACD model, the QACE model, and the PIEP method are $\sigma^{(1)} \simeq 7.0 \times 10^{-3} \AA, \sigma^{(2)} \simeq 6.5 \times 10^{-3} \AA^{2}, \sigma^{(3)} \simeq 8.1 \times 10^{-5} \AA^{3}$, and $\sigma^{(4)} \simeq 1,6 \times 10^{-6} \AA^{4}, \sigma^{(1)} \simeq 6.9 \times 10^{-3} \AA, \sigma^{(2)} \simeq 6.5 \times 10^{-3} \AA^{2}$, $\sigma^{(3)} \simeq 7.6 \times 10^{-5} \AA^{3}$, and $\sigma^{(4)} \simeq 1,4 \times 10^{-6} \AA^{4} \quad$ [34], and $\sigma^{(2)} \simeq 5.7 \times 10^{-3} \AA^{2}$ and $\sigma^{(3)} \simeq 7.7 \times 10^{-5} \AA^{3}$ [17], respectively, while the experimental values are $\sigma^{(1)} \approx 5.9 \times 10^{-3} \AA$, $\sigma^{(2)} \approx 6.8 \times 10^{-3} \AA^{2}, \sigma^{(3)} \approx 9.2 \times 10^{-5} \AA^{3}$, and $\sigma^{(4)} \approx 1,9 \times 10^{-6} \AA^{4}$ at $293 \mathrm{~K}[35], \sigma^{(2)} \approx 5.8 \times 10^{-3} \AA^{2}$ and $\sigma^{(3)} \approx 8.6 \times 10^{-5} \AA^{3}$ at $300 \mathrm{~K}[17]$.

Thus, the calculated results of the first four EXAFS cumulants of Ni by the QACE model are in agreement with those obtained using the other theoretical methods and experiments at various temperatures. It shows that the efficiency of the QACE model in calculating the EXAFS cumulants for metal crystals.
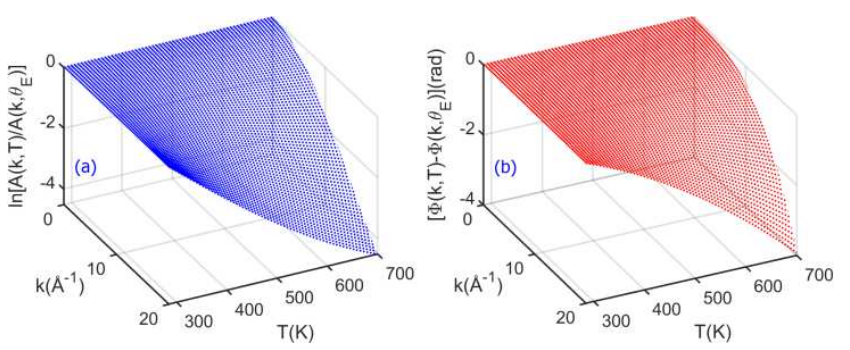

Figure 3. Wavenumber and temperature dependence of (a) the logarithm of amplitude ratio (blue mesh-surfaces) and (b) the phase difference (red meshsurfaces) with reference value at $\theta_{E}$ of Ni obtained using the QACE model.

The wavenumber and temperature dependence of (a) the logarithm of amplitude ratio $M(k, T)=\ln \left[A(k, T) / A\left(k, \theta_{E}\right)\right]$ and (b) the phase difference $\Delta \Phi(k, T)=\Phi(k, T)-\Phi\left(k, \theta_{E}\right)$ of $\mathrm{Ni}$ is calculated by Eqs. (2)-(3) and shown in Figure 2. Herein, our calculations used the QACE model to determine the EXAFS cumulants via Eqs. (9)-(12). It can be seen that the results of $M(k, T)$ and $\Delta \Phi(k, T)$ decrease with increasing temperature $T$ and decrease with fast-increasing wavenumber $k$. For example, at $T=400 \mathrm{~K}$, our calculated results are $M(k, T) \simeq-0.41$ and $\Delta \Phi(k, T) \simeq-0.09 \mathrm{rad}$ at $k=10 \AA^{-1}$, and $M(k, T) \simeq-1.48$ and $\Delta \Phi(k, T) \simeq-0.73 \mathrm{rad}$ at $k=20 \AA^{-1}$, while the corresponding results at $T=600 \mathrm{~K}$ are $M(k, T) \simeq-1.11$ and $\Delta \Phi(k, T) \simeq-0.32 \mathrm{rad}$ at $k=10 \AA^{-1}$, and $M(k, T) \simeq-3.66$ and $\Delta \Phi(k, T) \simeq-2.57 \mathrm{rad}$ at $k=20 \AA^{-1}$.

Thus, the cumulants are very important for the quantitative analysis of the anharmonic EXAFS spectra, in which the even-order cumulants contribute to the amplitude reduction, and the odd-order cumulants contribute primarily to the phase shift of the anharmonic EXAFS oscillation. Accurate calculation of the EAFS cumulants will allow us to accurately analyze the change of the anharmonic EXAFS spectra, and from which one will determine the structural parameters from the experimental EXAFS data.

\section{Conclusions}

In this work, the QACE model has been used successfully in the analysis of the anharmonic EXAFS spectra. The EXAFS analysis is performed based on evaluating the contribution of the DW factors to the amplitude reduction and phase shift in the cumulant expansion approach up to fourth-order. These analytical results show the role of the high-order EXAFS cumulants in the anharmonic EXAFS oscillation.

Although the QACE model uses a one-dimensional model, three-dimensional interactions were taken into account via a simple procedure. It derived from the anharmonic effective potential that includes all of the nearest-neighbor interactions of the absorbing and backscattering atoms.

The good agreement of our numerical results for $\mathrm{Ni}$ with those obtained using the ACD model, the PIEP method, and the other experiments at various temperatures show the effectiveness of the QACE model for calculating and analyzing the anharmonic EXAFS oscillation. These numerical results are very useful for analyzing the data of the anharmonic EXAFS spectra of the metal crystals.

\section{Acknowledgements}

This work was supported by the Institute of Research and Development, Duy Tan University, Danang, Vietnam.

\section{Author Contribution Statement}

We state that all authors contributed to the results of this work, where the revisions related to the comments of reviewers have been performed by the corresponding author Tong Sy Tien.

\section{Conflicts of Interest Statement}

All the authors do not have any possible conflicts of interest. 


\section{ORCID iDs}

Tong Sy Tien (iD http://orcid.org/0000-0002-7849-4456.

\section{References}

[1] F. W. Lytle, D. E. Sayers, and E. A. Stern, Phys. Rev. B 11, 4825 (1975).

[2] G. Beni and P. M. Platzman, Phys. Rev. B 14, 1514 (1976).

[3] P. Eisenberger and G. S. Brown, Solid State Commun. 29, 481 (1979).

[4] R. B. Greegor and F. W. Lytle, Phys. Rev. B 20, 4902 (1979).

[5] E. A. Stern, BA Bunker, and S. M. Heald, Phys. Rev. B 21, $5521(1980)$.

[6] P. A. Lee, P. H. Citrin, P. Eisenberger, and B. M. Kincaid, Rev. Mod. Phys. 53, 769 (1981).

[7] G. Bunker, Nucl. Instrum. Methods 207, 437 (1983).

[8] L. Tröger, T. Yokoyama, D. Arvanitis, T. Lederer, M. Tischer, and K. Baberschke, Phys. Rev. B 49, 888 (1994).

[9] G. Dalba, P. Fornasini, and M. Grazioli, Phys. Rev. B 76, 11034 (1995)

[10] J. J. Rehr and R. C. Albers, Rev. Mod. Phys. 72, 621 (2000).

[11] J. M. Tranquada and R. Ingalls, Phys. Rev. B 28, 3520 (1983).

[12] E. D. Crozier, J. J. Rehr, and R. Ingalls, X-ray Absorption: Principles, Applications, Techniques of EXAFS, SEXAFS and XANES, edited by D. C. Koningsberger \& R. Prins, John Wiley \& Sons, New York, 1988, Chap. 9.

[13] G. Dalba and P. Fornasini, J. Synchrotron Radiat. 4, 243 (1997).

[14] G. Dalba, P. Fornasini, R. Grisenti, D. Pasqualini, D. Diop, and F. Monti, Phys. Rev. B 58, 4793 (1998).

[15] N. V. Hung and J. J. Rehr, Phys. Rev. B 56, 43 (1997).

[16] T. Miyanaga and T. Fujikawa, J. Phys. Soc. Jpn. 63, 3683 (1994).

[17] T. Yokoyama, Phys. Rev. B 57, 3423 (1998).
[18] T. Yokoyama, J. Synchrotron Radiat. 6, 323 (1999).

[19] A. V. Poiarkova and J. J. Rehr, Phys. Rev. B 59, 948 (1999).

[20] S. a Beccara, G. Dalba, P. Fornasini, R. Grisenti, F. Pederiva, A. Sanson, D. Diop, and F. Rocca, Phys. Rev. B 68, 140301 (2003).

[21] S. a Beccara and P. Fornasini, Phys. Rev. B 77, 172304 (2008).

[22] F. D. Vila, J. J. Rehr, H. H. Rossner, and H. J. Krappe, Phys. Rev. B 76, 014301 (2007).

[23] F. D. Vila, V. E. Lindahl, and J. J. Rehr, Phys. Rev. B 85, 024303 (2012).

[24] A. Sanson, J. Synchrotron Radiat. 16, 864 (2009).

[25] T. S. Tien, J. Phys. D: Appl. Phys. 53 (2020) 315303.

[26] N. V. Hung, L. H. Hung, T. S. Tien, and R. R. Frahm, Int. J. Mod. Phys. B 22, 5155 (2008).

[27] N. V. Hung, C. S. Thang, N. B. Duc, DQ. Vuong, and T. S. Tien, Eur. Phys. J. B 90, 256 (2017).

[28] N. V. Hung, N. B. Duc, DQ. Vuong, N. C. Toan, and T. S. Tien, Vacuum 169, 108872 (2019).

[29] N. V. Hung, T. S. Tien, N. B. Duc, and DQ. Vuong, Mod. Phys. Lett. B 28, 1450174 (2014).

[30] T. S. Tien, N. V. Hung, N. T. Tuan, N. V. Nam, N. Q. An, N. T. M Thuy, V. T. K. Lien, and N. V. Nghia, J. Phys. Chem. Solids 134, 307 (2019).

[31] L. A. Girifalco and V. G. Weizer, Phys. Rev. 114, 687 (1959).

[32] T. Fujikawa and T. Miyanaga, J. Phys. Soc. Jpn. 62, 4108 (1993).

[33] T. Yokoyama, K. Kobayashi, T. Ohta, and A. Ugawa, Phys. Rev. B 53, 6111 (1996).

[34] N. V. Hung, N. B. Trung, and N. B. Duc, J. Mater. Sci. Appl. $1,51(2015)$.

[35] I. V. Pirog, T. I. Nedoseikina, I. A. Zarubin, and A. T. Shuvaev, J. Phys.: Condens. Matter 14, 1825 (2002). 